\title{
On the reliability of computation of maximum Lyapunov Characteristic Exponents for asteroids
}

\author{
Zoran Knežević and Slobodan Ninković \\ Astronomical Observatory, Volgina 7, 11160 Belgrade 74, Serbia and Montenegro \\ email: zoran@aob.bg.ac.yu; sninkovic@aob.bg.ac.yu
}

\begin{abstract}
We present an analysis of the reliability of computation of maximum Characteristic Exponents of Lyapunov from the numerical integrations of asteroid orbits over finite intervals of time. We used two complementary approaches - a comparison of the LCE estimates from the backward and forward integrations of orbits, and a comparison of the estimates coming from the integrations of the same initial conditions over different time spans. The main conclusion is that for a vast majority of asteroids $(>80 \%)$ the results can be considered as reliable enough to reveal the very nature and basic properties of the motion.
\end{abstract}

Keywords. Asteroids, chaotic dynamics, maximum Lyapunov Characteristic Exponents

\section{Introduction}

Chaotic dynamics in the real systems can be quite complex, thus giving rise to very different diffusion times and macroscopic effects. In order to assess the stochastic motion, to identify and quantitatively describe the chaos, one usually computes some sort of indicator or measure, which reveals the very character of the motion, or distinguishes between phenomena related to the chaotic dynamics.

The most commonly used measure of chaos is the so-called maximum Lyapunov Characteristic Exponent (LCE). It measures the rate at which two initially nearby orbits diverge with respect to each other, in terms of some convenient metrics in the phase space of state vectors (Milani \& Mazzini 1997). The stronger the chaos, the faster the divergence and the larger the LCE, so that a large, positive LCE typically means that the corresponding orbit is strongly chaotic.

By definition, Lyapunov exponents are all the real numbers obtained from a limit:

$$
\chi\left(X_{0}, V_{0}\right)=\lim _{t \rightarrow \infty} \frac{\gamma(t)}{t} ; \quad \gamma(t)=\log _{e} \frac{|V(t)|}{\left|V_{0}\right|} .
$$

Given the dynamical system $\dot{X}=F(X)$ and its general solution $\Phi^{t}\left(X_{0}\right)=X(t)$ in the form of an integral flow, $V(t)$ is found as a solution (with initial condition $V_{0}$ ) of a variational equation (with respect to the orbit with initial condition $X_{0}$ ):

$$
\dot{V}=\frac{\partial F}{\partial X}(X(t)) V
$$

associated with the dynamical system.

In practice, however, we are more often using the Lyapunov time $T_{L}=1 / \chi$, which is defined as a simple inverse of the LCE. Lyapunov time is easier to comprehend and interpret, in particular when discussing the dynamical mechanisms at work, or when we 
are to compare the chaotic behavior of different bodies. Obviously, $T_{L}$ represents the time needed to increase the distance between the orbits $\exp (1)$ times.

Many alternative methods to identify and quantify chaos and to distinguish between phenomena related to chaotic dynamics - invariant tori, islands of libration, chaotic zones, etc., have been proposed over the last 15 years, like the frequency map analysis and the related sup-map analysis (Laskar 1990; 1993), the short-time measures like the local Lyapunov characteristic numbers (Froeschlé et al. 1993), or stretching numbers (Voglis \& Contopoulos 1994), helicity and twist angles, azimuthal and rotation angles (Contopoulos et al. 1997), the so-called fast Lyapunov indicators (Froeschlé et al. 1998), etc. A comprehensive review of various methods can be found in Knežević (2000).

For the purpose of this paper, however, we stick with the LCE only. In Section 2 we describe the specific problem we are dealing with and the methods used to tackle it, in Section 3 we give and discuss the results, and in Section 4 we offer some conclusions.

\section{Computation of maximum LCEs for asteroids}

In the case of solar system bodies, like planets or asteroids, the dynamical system is well-known to be non integrable, so that an exact computation of LCE for $t \rightarrow \infty$ is not possible. The simplest way to get an approximate, temporary estimate of the value of maximum LCE is by monitoring the variation of $\gamma(t)$ with time, computed on-line, in the course of the numerical integration of the orbit itself; if this variation has an asymptotic character, the slope of the variation gives the estimated value of the maximum LCE.

In order to compute an estimate of the maximum LCE reliably, one has to integrate the orbit for at least 6-10 times $T_{L}$ or even longer (Froeschlé et al. 1998); in the case of weak, slow chaos this can become quite a formidable task and a very time-consuming procedure. On the other hand, a single integration of a strongly chaotic orbit does not posses any predictive value; only a large ensemble of integrations with the same or similar initial conditions, integration methods and platforms can supply us with a valid indication on the nature and characteristics of the chaotic motion; again this is a time-consuming procedure, applicable only in some special cases.

For authors of asteroid catalogs which include hundreds of thousands of bodies, where LCEs are provided with other orbital information, the essential becomes to know a priori whether the effort of computing all these estimates is really meaningful, that is, whether the Lyapunov Characteristic Exponents can at all be reliably determined from the integrations covering only a limited time span. For users of these catalogs the question is whether they can believe and apply these estimates at face value, or, at least, what is the probability that the computed values are indeed indicative of the true nature of motion.

We used in our analysis the data obtained as part of the regular updates of the AstDyS databaset, but also a large sample of integrations adapted to and carried out specifically for the purpose of this study.

We employed two different, but complementary approaches: (i) the comparison of the LCEs derived separately from two integrations, starting from the same initial conditions, using the same method, code and machines, and covering the same interval of time with the same step size, but once with the negative step (that is, propagating orbit into the past) and the other time with the positive step (propagating into the future); (ii) the comparison of the LCEs derived from the integrations starting again from the same initial initial conditions, using the same method, code and machines, but this time covering different intervals of time and integrating either forward or backward in time.

$\dagger$ http://hamilton.dm.unipi.it/astdys 


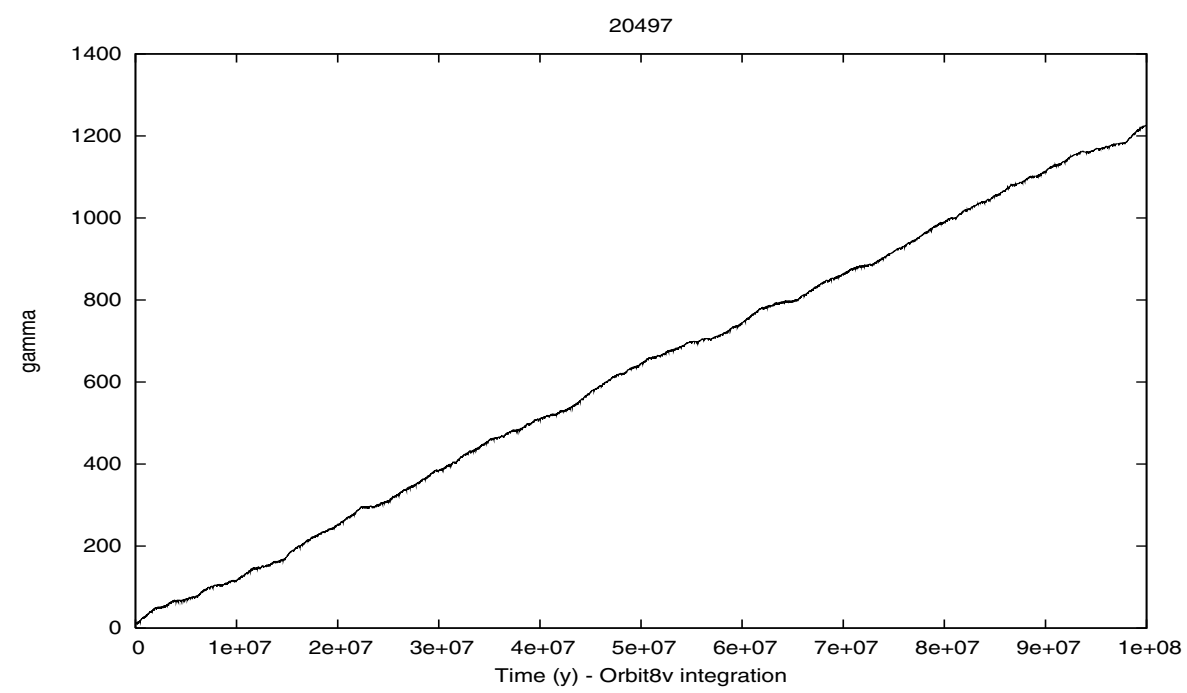

Figure 1. Asteroid 20497. A smooth variation of $\gamma(t)$. Maximum LCE can be estimated in a reliable manner. Orbit is moderately chaotic with $T_{L} \approx 83000 \mathrm{yr}$.

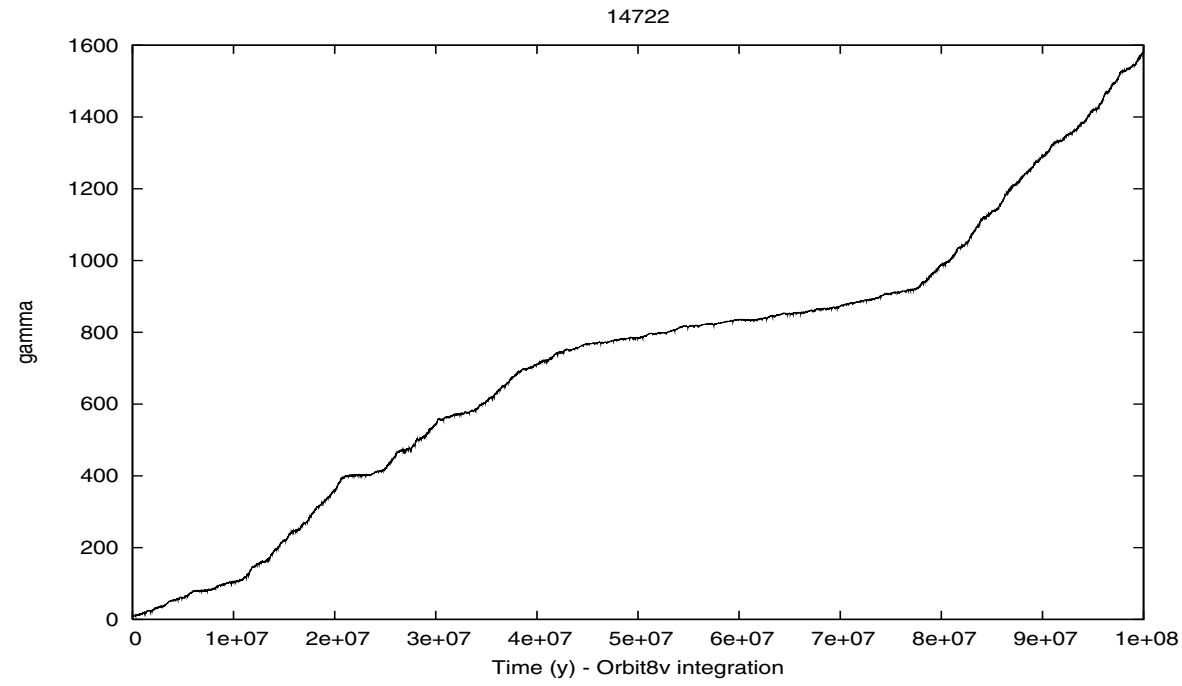

Figure 2. Maximum LCE estimate for asteroid 14722. Corresponding Lyapunov time is $T_{L} \approx 75000$ yr. Note abrupt changes of the slope of $\gamma(t)$, including episodes of strong chaos $\left(T_{L} \approx 26000 \mathrm{yr}\right)$. This orbit passes through different dynamical regimes in the course of time, and the estimate of the corresponding LCE in this case critically depends on the integration time span.

In the first set of experiments we in fact varied the configuration of the dynamical system and thus the circumstances of the determination of corresponding LCEs. We used integrations of asteroid orbits covering $1 \mathrm{Myr}$ in the past and $1 \mathrm{Myr}$ in the future. The LCEs were determined from the two integrations separately and then simply compared, assuming the values to be the same if the difference was less than $10^{-5} \mathrm{yr}^{-1}$ (this values corresponds to $T_{L}>100000 \mathrm{yr}$, i.e. to a somewhat conservative requirement that for reliable computation of LCEs we need integrations covering at least $10 T_{L}$ ). 

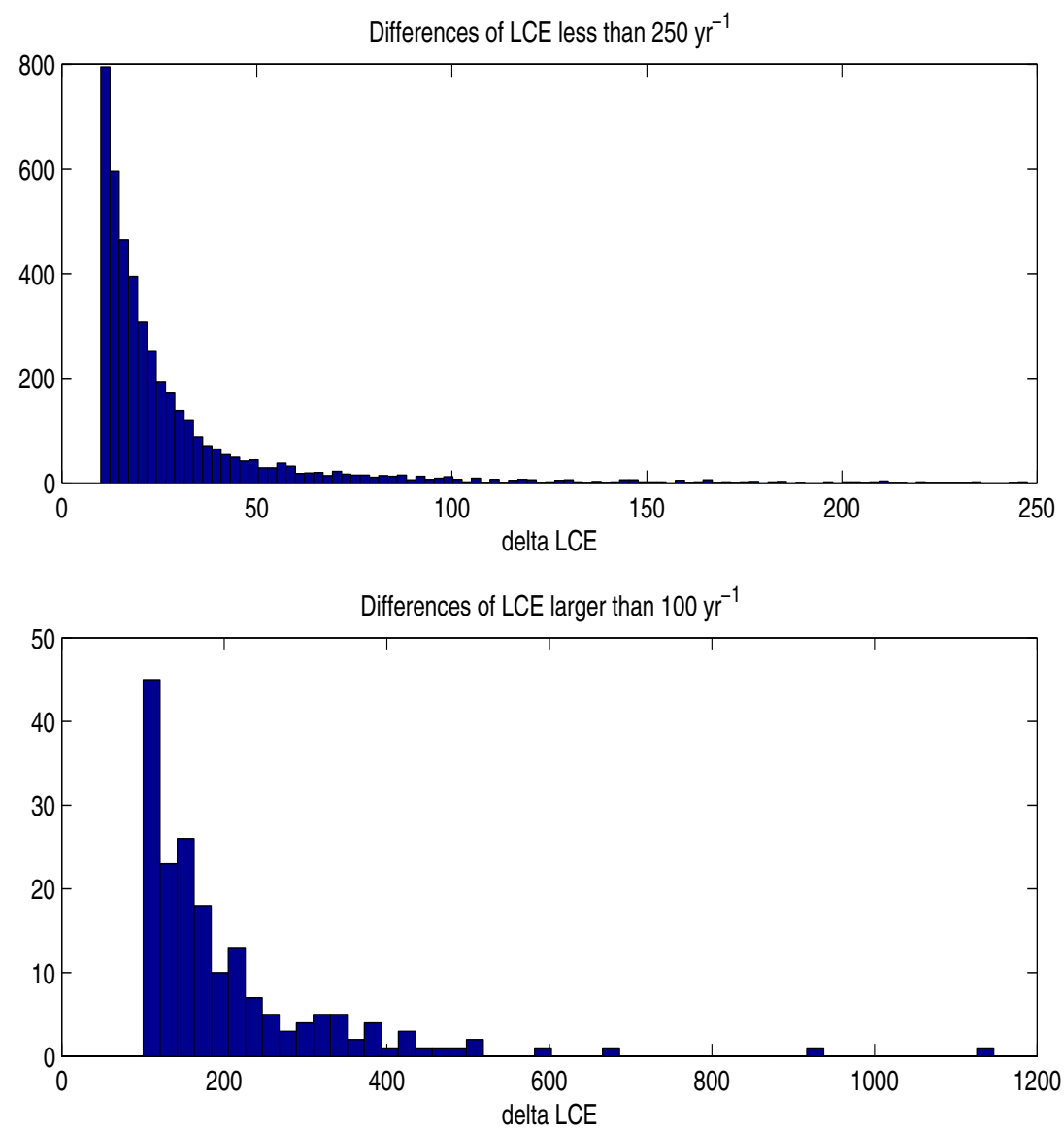

Figure 3. Number frequency distributions of differences of maximum LCEs in units of $10^{-6}$. Above: asteroids with differences $<250 \mathrm{yr}^{-1}$. Below: the large differences tail of the distribution.

In the second set of experiments we were repeating computations over intervals of time spanning the two orders of magnitude. More precisely, we compared the outcomes of integrations covering 2, 10 and $100 \mathrm{Myr}$. The problem we are dealing with in this case is illustrated with the examples of the computation of $\gamma(t)$ given in Figs. 1 and 2.

\section{Results}

All the computations in this study were performed by using the public domain software packages OrbFit and Orbit9, available from the aforementioned AstDyS site. In particular, we made use of the Orbit9e integrator and of the software to compute synthetic proper elements, described in more detail in Knežević \& Milani (2000).

Summarizing the results of the first set of experiments, let us repeat that we compared two estimates of the maximum LCEs obtained from the forward and backward $1 \mathrm{Myr}$ integrations for a total of 23767 asteroids. Out of these, for 19371 (or 81.5\%) the differences of LCEs were $\leqslant 10^{-5} \mathrm{yr}^{-1}$, that is below the adopted accuracy threshold for reliable LCE computation. Thus, for the vast majority of asteroids the results derived from two integrations can be considered the same and safely used as indicator of the true nature of motion. 

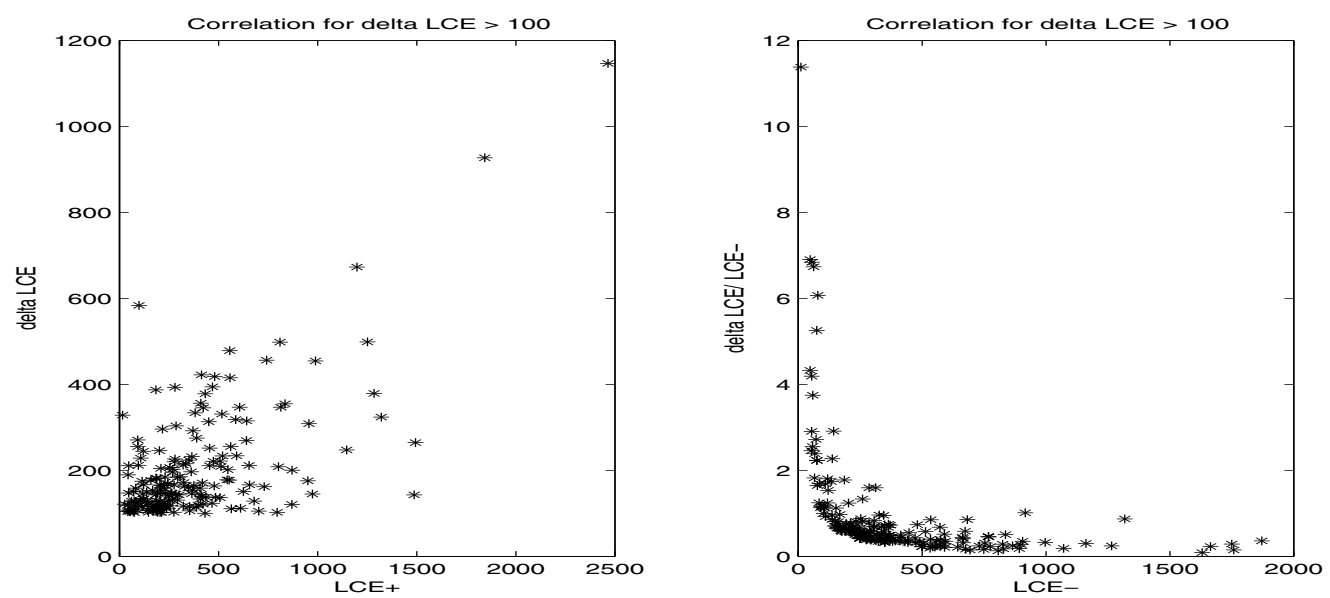

Figure 4. Differences of maximum LCE vs. one of the estimates for 183 objects with largest LCE differences (left). Relative difference vs. the other estimate for the same objects (right). All quantities are in units of $10^{-6}$.

Of particular interest are, however, objects for which large differences of LCE estimates were found. There were 4396 asteroids with difference $>10^{-5} \mathrm{yr}^{-1}$ (the number frequency distribution of the differences of maximum LCEs up to $2.5 \times 10^{-4} \mathrm{yr}^{-1}$ is given in the upper panel of Fig. 3), out of which 183 with difference $>10^{-4} \mathrm{yr}^{-1}$ (see the distribution in lower panel of Fig. 3), and 40 with difference $>2.5 \times 10^{-4} \mathrm{yr}^{-1}$. The median difference for all 4396 asteroids with LCE differences above the accuracy threshold is found to be $\approx 1.9 \times 10^{-5} \mathrm{yr}^{-1}$. Thus, even if the maximum LCEs for these bodies have been estimated with lower accuracy, there is still a good fraction of objects for which this estimate reliably reflects at least the very nature of the motion (e.g. strongly vs. moderately chaotic).

In order to identify the "troublemakers", in Fig. 4 we plotted the differences of the estimates against the estimates themselves, both in absolute and relative terms. We expected to find correlation in the sense - more chaotic the motion, less reliable the computation of the corresponding LCE; this sort of correlation appears quite obvious in the left panel, showing the absolute values of the differences vs. differences themselves. The result in the right hand side panel, showing the relative differences vs. differences themselves, indicates, however, that comparatively more important appear to be differences for the moderately chaotic orbits. For the asteroids with such differences computation of the maximum LCE is unreliable and the obtained values cannot be considered useful.

In Table 1 we give asteroids from our sample of 183 bodies with significant differences of LCE that have largest absolute and relative differences of the computed LCEs. In the first three columns we list asteroid number, maximum LCE as computed from the forward integration, and the corresponding LCE difference for 12 asteroids for which this difference was found to be larger then $4 \times 10^{-4} \mathrm{yr}^{-1}$. In the remaining three columns we show again asteroid number, but with maximum LCE as computed from the backward integration, and the corresponding relative LCE difference for 9 asteroids for which this difference was larger then 3 .

Repeating now more-or-less the same exercise for the second set of experiments, we begin with comparison of the LCEs computed from the integrations covering 2 and 10 Myr. Let us emphasize that asteroids for which we extend integrations from 2 to $10 \mathrm{Myr}$ in the regular AstDyS database updates are those for which some sort of problem has been detected in the shorter integration; typically this might have been either the 
Table 1. Asteroids from our sample of 183 bodies with largest absolute and relative differences of the computed LCEs

\begin{tabular}{cccccr}
\hline Asteroid & $\begin{array}{c}L C E+ \\
\times 10^{-6} \mathrm{yr}^{-1}\end{array}$ & $\begin{array}{c}\text { Abs. Diff } \\
\times 10^{-6} \mathrm{yr}^{-1}\end{array}$ & Asteroid & $\begin{array}{c}L C E- \\
\times 10^{-6} \mathrm{yr}^{-1}\end{array}$ & Rel. Diff \\
\hline 22541 & 1198 & 673 & 22269 & 29 & 4.2 \\
29180 & 480 & 418 & 22272 & 18 & 6.7 \\
37152 & 742 & 456 & 22378 & 68 & 6.0 \\
42485 & 557 & 478 & 22383 & 65 & 11.4 \\
42600 & 415 & 422 & 22467 & 10 & 6.8 \\
43946 & 558 & 415 & 22502 & 10 & 6.9 \\
49737 & 989 & 455 & 22543 & 37 & 3.7 \\
53463 & 1844 & 927 & 22553 & 10 & 4.3 \\
58949 & 2465 & 1147 & 22582 & 13 & 5.3 \\
72205 & 811 & 498 & & & \\
75246 & 1251 & 499 & & & \\
80143 & 99 & 584 & & & \\
\hline
\end{tabular}

poor accuracy of the resulting synthetic proper elements, or large positive LCE indicating strongly chaotic orbit. It is thus no surprise that out of a total of 5768 asteroids considered in the second set of experiments, in only 3663 , or $63.5 \%$ of the cases, we found good agreement of the estimates of maximum LCE from the two integrations. In the remaining 2105 cases the difference was larger than $5 \times 10^{-6} \mathrm{yr}^{-1}$, which was in this case the accuracy threshold for the shorter integration. Nevertheless, the number of significantly different estimates was not very high, just 113 objects with differences $>10^{-4} \mathrm{yr}^{-1}$ and 17 objects with differences $>2.5 \times 10^{-4} \mathrm{yr}^{-1}$; median for objects with difference above the accuracy threshold was $\approx 2.2 \times 10^{-5} \mathrm{yr}^{-1}$. Fig. 5 , analogous with Fig. 3, shows the same number frequency distributions as in the previous case.

The most interesting in this context are, however, the results of comparison with integrations covering 100 Myr time span. Here we compared our estimates of LCE for a specially selected group of asteroids belonging to Veritas asteroid family; these asteroids exhibit very different dynamical behaviors, ranging from very stable to strongly chaotic (Knežević et al. 2002). We collected all the interesting results in Table 2. We mixed different integrations here, in order to check all possible outcomes and to partly compensate for a small number of experiments performed in this case. Thus we compare estimates of maximum LCE from short integrations, covering either 2 or $10 \mathrm{Myr}$, with $100 \mathrm{Myr}$ forward or backward integrations. As one can easily see, even under these "extreme" circumstances the results in most cases agree quite well (note that in columns 4 and 5 all the printed figures are significant). In each of the several cases in which the agreement was found not to be that good, the comment "chs" indicates that the body changed the dynamical state during the interval of time covered by the integration, and that therefore we detected a significant change of slope of $\gamma(t)$ like in the example shown in Fig. 2.

\section{Conclusions}

We have conducted a comprehensive analysis of the reliability of computation of maximum Characteristic Exponents of Lyapunov from the numerical integrations of asteroid orbits covering finite intervals of time. We used two complementary approaches - a comparison of the results coming from the backward and forward integrations of orbits, and similar comparison of the results coming from the integrations of the same initial conditions, but over very different time spans. 

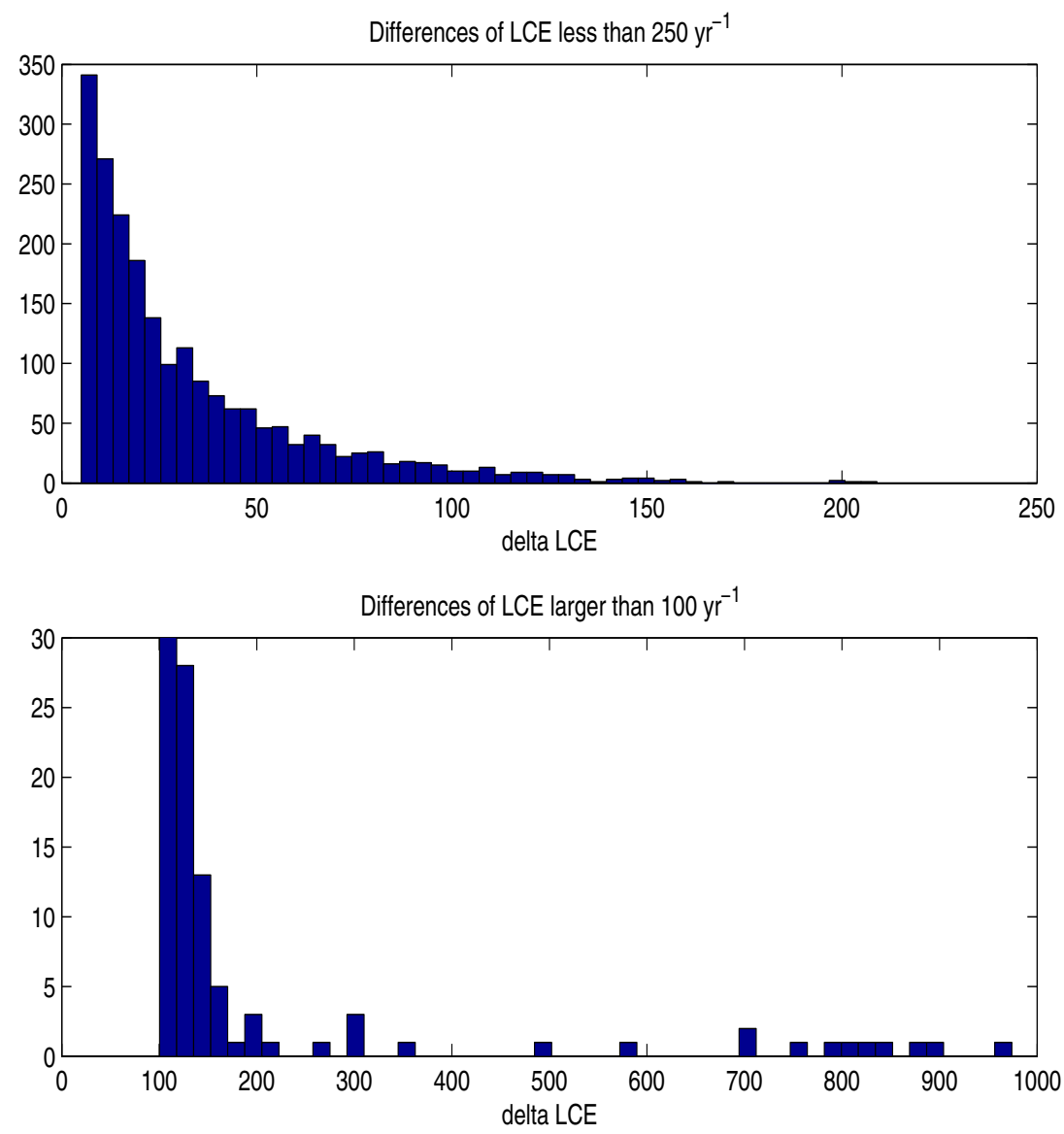

Figure 5. Number frequency distributions of differences of maximum LCEs in units of $10^{-6}$ obtained from integrations covering 2 and $10 \mathrm{Myr}$. Above: asteroids with differences $<250 \mathrm{yr}^{-1}$. Below: the large differences tail of the distribution.

The main conclusion which can be drawn from this analysis is that for a vast majority of asteroids $(>80 \%)$ the results of the computation, even if stochastic by definition, can be considered as reliable enough to reveal the very nature and basic properties of the motion. For chaotic bodies the LCE estimates are also in most cases $(>60 \%)$ precise enough at least to clearly distinguish between different dynamical regimes (weak to moderate vs. strong chaos). Still, we recommend to always take the computed values of LCEs with some caution, in particular when dealing with outcomes of short integrations or with strongly chaotic motion. Even in the case the computed values indicate very stable motion, they should not be taken uncritically and at face value, but always bearing in mind their probabilistic definition and interpretation.

\section{Acknowledgements}

We would like to acknowledge the contribution of A. Milani, the principal author of the software packages OrbFit and Orbit9 which we used for all the computations. This research is supported by the Ministry of Science and Environmental Protection of Serbia through project No 1238. 
Table 2. Asteroids in Veritas asteroid family exhibit very different dynamical behavior - from very stable to strongly chaotic. In the first column we give asteroid number, maximum LCE in the second column was obtained from the integration covering a time span indicated in the third column (negative time refers to the backward integration), LCEs as obtained from the forward and backward integrations are shown in the fourth and fifth columns, respectively; the comment is added in the final column ("chs" stands for the "changing state").

\begin{tabular}{|c|c|c|c|c|c|}
\hline Asteroid & $\begin{array}{c}\mathrm{LCE} \\
\times 10^{-6} \mathrm{yr}^{-1}\end{array}$ & $\begin{array}{l}\Delta T \\
\mathrm{Myr}\end{array}$ & $\begin{array}{c}\mathrm{LCE}+100 \\
\times 10^{-6} \mathrm{yr}^{-1}\end{array}$ & $\begin{array}{c}\text { LCE- } 100 \\
\times 10^{-6} \mathrm{yr}^{-1}\end{array}$ & Comment \\
\hline 490 & 98 & 10 & & 112.8 & \\
\hline 1086 & 5 & -2 & 0.2 & & \\
\hline 2147 & 7 & -2 & 8.1 & & \\
\hline 2428 & 5 & -2 & 2.9 & & \\
\hline 2934 & 5 & -2 & 3.7 & & \\
\hline 3090 & 5 & -2 & 0.6 & & \\
\hline 3542 & 105 & 10 & & 107.7 & \\
\hline 5594 & 29 & -2 & 22.6 & & \\
\hline 6374 & 5 & -2 & 0.0 & & \\
\hline 7231 & 5 & -2 & 0.0 & & \\
\hline 7612 & 30 & -2 & 18.6 & & \\
\hline 7626 & 5 & -2 & 4.5 & & \\
\hline 8726 & 107 & 10 & & 98.5 & \\
\hline 9715 & 5 & -2 & 0.6 & & \\
\hline 10120 & 114 & 10 & & 91.9 & \\
\hline 10414 & 5 & -2 & 0.9 & & \\
\hline 10793 & 37 & -2 & 22.5 & & \\
\hline 13537 & 12 & -2 & 11.1 & & \\
\hline 14722 & 39 & -2 & 13.2 & & \\
\hline 19280 & 31 & -2 & 20.1 & & \\
\hline 20497 & 9 & -2 & 12.2 & & \\
\hline 21454 & 98 & 10 & & 93.2 & \\
\hline 24718 & 105 & 10 & & 79.5 & chs \\
\hline 32416 & 95 & 10 & & 91.8 & \\
\hline 38447 & 112 & 10 & & 103.0 & \\
\hline 39290 & 108 & 10 & & 111.2 & \\
\hline 42218 & 110 & 10 & & 106.4 & \\
\hline 43095 & 35 & 10 & & 102.2 & chs \\
\hline 45623 & 106 & 10 & & 103.0 & \\
\hline 46105 & 110 & 10 & & 82.9 & chs \\
\hline 51136 & 104 & 10 & & 14.9 & chs \\
\hline 54869 & 108 & 10 & & 93.0 & chs \\
\hline
\end{tabular}

\section{References}

Contopoulos, G., Voglis, N., Efthymiopoulos, C., Froeschl, C., Gonczi, R., Lega, E., Dvorak, R., \& Lohinger, E. 1997, Cel. Mech. Dyn. Astron. 67, 293

Froeschlé, C., Froeschlé, Ch., \& Lohinger, E. 1993, Cel. Mech. Dyn. Astron. 56, 307

Froeschlé, C., Gonczi, R., Lega, E., \& Locatelli, U. 1998, Cel. Mech. Dyn. Astron. 69, 235

Knežević Z. 2000, in: N. Bokan (ed.) Proceedings Symp. Contemp. Math.: 125 years of Faculty of Mathematics, Fac. Math. Univ. Belgrade, p. 269

Knežević Z., \& Milani A. 2000, Cel. Mech. Dyn. Astron. 78, 17

Knežević Z., Tsiganis K., \& Varvoglis H. 2002, in Asteroids, Comets, Meteors ACM2002., (European Space Agency SP-500), 335.

Laskar, J. 1990, Icarus 88, 266

Laskar, J. 1993, Physica D 67, 257

Milani, A. \& Mazzini, G. 1997, Sistemi dinamici (Pisa University Editorial Service)

Voglis, N. \& Contopoulos, G. J. 1994, J. Phys. A: Math. Gen. 27, 4899 